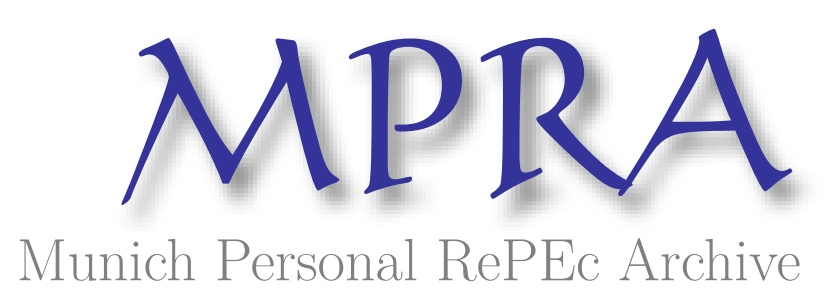

\title{
On multi-particle Brownian survivals and the spherical Laplacian
}

B S, Balakrishna

4 January 2013

Online at https://mpra.ub.uni-muenchen.de/43567/

MPRA Paper No. 43567, posted 04 Jan 2013 16:49 UTC 


\title{
On Multi-Particle Brownian Survivals and the Spherical Laplacian
}

\author{
B S Balakrishna*
}

January 4, 2013

\begin{abstract}
The probability density function for survivals, that is for transitions without hitting the barrier, for a collection of particles driven by correlated Brownian motions is analyzed. The analysis is known to lead one to a study of the spectrum of the Laplacian on domains on the sphere in higher dimensions. The first eigenvalue of the Laplacian governs the large time behavior of the probability density function and the asymptotics of the hitting time distribution. It is found that the solution leads one naturally to a generating function for the eigenvalues and multiplicities of the Laplacian. Analytical properties of the generating function suggest a simple scaling procedure for determining the eigenvalues readily applicable for a homogeneous collection correlated particles. Comparison of the first eigenvalue with the theoretical and numerical results of Ratzkin and Treibergs for some special domains shows remarkable agreement.
\end{abstract}

The case of a particle obeying Brownian motion in one dimension under different boundary conditions have been well studied. For instance, in the simplest case of a single barrier, the probability density function for transition without hitting the barrier is expressible in closed form. No closed form solutions exist in the case of a collection of such particles driven by correlated Brownian motions. The problem of $n$ particles can be recast into a that of solving the heat equation or the diffusion equation in a conical region in $n$-dimensions. Within such a context, the problem has been addressed by various authors in the past and series solutions have been obtained expressible in terms of modified Bessel functions. The $n=2$ solution was obtained by Sommerfeld [1894]. It has been addressed within the context of default correlation by Zhou [2001]. The $n=3$ case was considered within the context of circular cones by Carslaw and Jaeger [1959]. For higher dimensions, the applicable solution has been presented by Cheeger [1983]. The probability of survival as such was obtained by DeBlassie [1987] and its implications for hitting times discussed.

The problem of $n$ Brownian particles restricted by barriers leads to a heat equation in $n$ dimensions. The radial component of the equation is identifiable with the differential equation for a Bessel process whose solution is well-known. The angular component of the series solution involves the eigenvalues and the eigenfunctions of the Laplacian for a domain on the $n-1$ dimensional sphere. The first eigenvalue of the Laplacian determines the large time behavior of the survival probability and hence the finiteness of the expected hitting time. It is found that the solution leads naturally to a generating function for the eigenvalues and their multiplicities. Analytical properties of the generating function suggest a simple scaling procedure to estimate the other eigenvalues, easily applicable to

*balakbs2@gmail.com 
a homogeneous collection of correlated particles. The estimate to the first eigenvalue finds excellent agreement with the theoretical and numerical results obtained by Ratzkin and Treibergs [2009] for some special domains within the context of a capture problem.

The article is organized as follows. Sections 1, 2 and 3 address the solutions for one, two and many particle systems. Section 4 discusses a generating function for the spectrum of the Laplacian arising naturally from the series solution. Section 5 analyzes some of the interesting analytical properties of the generating function. Section 6 discusses a scaling procedure to estimate the eigenvalues and their applicability to a homogeneous collection of correlated particles. Section 7 compares the scaling procedure estimates with some of the available theoretical and numerical results.

\section{One Particle}

Consider a particle driven by Brownian motion with position variable $x$. The probability density $f\left(x, x^{\prime}, \tau\right)$ that the particle at position $x$ at any time $t$ reaches $x^{\prime}$ at time $T=t+\tau$ is obtained by solving the differential equation

$$
\frac{\partial f}{\partial \tau}=\frac{1}{2} \frac{\partial^{2} f}{\partial x^{2}}
$$

A constant drift term may also be present but ignored for simplicity of presentation. A suitable scaling of $x$ is done to standardize the coefficient of the second order term. The above is the heat equation or the diffusion equation having the fundamental solution

$$
f\left(x, x^{\prime}, \tau\right)=\frac{1}{\sqrt{2 \pi \tau}} e^{-\frac{1}{2 \tau}\left(x-x^{\prime}\right)^{2}} .
$$

As required, $f\left(x, x^{\prime}, \tau\right) \rightarrow \delta\left(x-x^{\prime}\right)$ as $\tau \rightarrow 0$.

Consider next a barrier at $x=0$. We will now be interested in the probability density that the particle at $x>0$ at any time $t$ reaches $x^{\prime}>0$ at time $T=t+\tau$ without hitting the barrier. The requirement that the particle does not hit the barrier at zero can be stated as a boundary condition $f\left(0, x^{\prime}, \tau\right)=0$. The solution to the differential equation is easily obtained by the method of images,

$$
f\left(x, x^{\prime}, \tau\right)=\frac{1}{\sqrt{2 \pi \tau}}\left(e^{-\frac{1}{2 \tau}\left(x-x^{\prime}\right)^{2}}-e^{-\frac{1}{2 \tau}\left(x+x^{\prime}\right)^{2}}\right)=\sqrt{\frac{2}{\pi \tau}} e^{-\frac{1}{2 \tau}\left(x^{2}+x^{\prime 2}\right)} \sinh \left(\frac{x x^{\prime}}{\tau}\right) .
$$

The total probability that the particle travels without hitting the barrier is then

$$
\int_{0}^{\infty} d x^{\prime} f\left(x, x^{\prime}, \tau\right)=1-2 N\left(-\frac{x}{\sqrt{\tau}}\right)
$$

where $N$ is the cumulative standard normal distribution function.

\section{Two Particles}

Next consider two particles with positions $x_{1}$ and $x_{2}$, together denoted $\boldsymbol{x}$, driven by Brownian motions correlated with a correlation parameter $\rho$. Let the barriers be set at $x_{1}=0$ and 
$x_{2}=0$ with boundary conditions $\left.f\left(\boldsymbol{x}, \boldsymbol{x}^{\prime}, \tau\right)\right|_{x_{1}=0}=\left.f\left(\boldsymbol{x}, \boldsymbol{x}^{\prime}, \tau\right)\right|_{x_{2}=0}=0$. The transition probability density $\frac{1}{\sqrt{1-\rho^{2}}} f\left(\boldsymbol{x}, \boldsymbol{x}^{\prime}, \tau\right)$ is now governed by the differential equation

$$
\frac{\partial f}{\partial \tau}=\frac{1}{2}\left[\frac{\partial^{2} f}{\partial x_{1}^{2}}+2 \rho \frac{\partial^{2} f}{\partial x_{1} \partial x_{2}}+\frac{\partial^{2} f}{\partial x_{2}^{2}}\right] .
$$

As before, for simplicity of presentation, constant drift terms are ignored and a suitable scaling of $x_{1}$ and $x_{2}$ is done to standardize the coefficients. The above equation can be diagonalized with the change of variables

$$
y_{1}=\frac{1}{\sqrt{1-\rho^{2}}}\left(x_{1}-\rho x_{2}\right), \quad y_{2}=x_{2} .
$$

In this new system of coordinates, the differential equation becomes

$$
\frac{\partial f}{\partial \tau}=\frac{1}{2}\left[\frac{\partial^{2} f}{\partial y_{1}^{2}}+\frac{\partial^{2} f}{\partial y_{2}^{2}}\right]
$$

This is the heat equation or the diffusion equation in 2-dimensions. Boundary conditions in the new coordinate system are

$$
x_{1}=0 \rightarrow y_{1}=-\frac{\rho}{\sqrt{1-\rho^{2}}} y_{2}, \quad x_{2}=0 \rightarrow y_{2}=0 .
$$

It is convenient to go to polar coordinates $r$ and $\theta$ where

$$
r=\sqrt{y_{1}^{2}+y_{2}^{2}}, \quad \theta=\cos ^{-1}\left(\frac{y_{1}}{r}\right), \quad 0 \leq \theta \leq \varphi=\cos ^{-1}(-\rho) .
$$

The differential equation to be solved now reads

$$
\frac{\partial f}{\partial \tau}=\frac{1}{2}\left[\frac{\partial^{2} f}{\partial r^{2}}+\frac{1}{r} \frac{\partial f}{\partial r}+\frac{1}{r^{2}} \frac{\partial^{2} f}{\partial \theta^{2}}\right] .
$$

Angular functions $\sin (\nu \theta)$ can be chosen to vanish on the boundaries at $\theta=0$ and $\theta=\varphi$ so that $f\left(\boldsymbol{x}, \boldsymbol{x}^{\prime}, \tau\right)$ can be expanded into Fourier series as

$$
f\left(\boldsymbol{x}, \boldsymbol{x}^{\prime}, \tau\right)=\sum_{\nu} g_{\nu}(r, \tau) r^{\nu} \sin (\nu \theta), \quad \nu=\frac{k \pi}{\varphi}, \quad k=1,2, \cdots
$$

The differential equation now reduces to

$$
\frac{\partial g_{\nu}}{\partial \tau}=\frac{1}{2} \frac{\partial^{2} g_{\nu}}{\partial r^{2}}+\frac{2 \nu+1}{2 r} \frac{\partial g_{\nu}}{\partial r}
$$

This is the differential equation describing the Bessel process. Its solution is well-known. $\frac{r^{\prime 2}}{\tau}$ is distributed as the non-central chi-squared distribution with $2(\nu+1)$ degrees of freedom and non-centrality parameter $\frac{r^{2}}{\tau}$. We thus have for the $r^{\prime}$-distribution

$$
g_{\nu}(r, \tau) \propto \frac{1}{\tau} \chi^{2}\left(\frac{r^{\prime 2}}{\tau}, 2(\nu+1), \frac{r^{2}}{\tau}\right) \propto \frac{1}{\tau} r^{-\nu} e^{-\frac{1}{2 \tau}\left(r^{2}+r^{\prime 2}\right)} I_{\nu}\left(\frac{r r^{\prime}}{\tau}\right)
$$


where $I_{\nu}$ is the modified Bessel function. Some factors involving $r^{\prime}$ have been dropped as the appropriate normalization is determined below. Putting together, we have

$$
f\left(\boldsymbol{x}, \boldsymbol{x}^{\prime}, \tau\right)=\frac{2}{\varphi \tau} e^{-\frac{1}{2 \tau}\left(r^{2}+r^{\prime 2}\right)} \sum_{\nu} I_{\nu}\left(\frac{r r^{\prime}}{\tau}\right) \sin (\nu \theta) \sin \left(\nu \theta^{\prime}\right) .
$$

To verify the factors, note that $d x_{1} d x_{2}=\sqrt{1-\rho^{2}} r d r d \theta$, and that $f\left(\boldsymbol{x}, \boldsymbol{x}^{\prime}, t\right) \rightarrow \delta(r-$ $\left.r^{\prime}\right) \delta\left(\theta-\theta^{\prime}\right)=\sqrt{1-\rho^{2}} \delta\left(x_{1}-x_{1}^{\prime}\right) \delta\left(x_{2}-x_{2}^{\prime}\right)$ in the limit $\tau \rightarrow 0$. The asymptotic behavior $I_{\nu}(x) \rightarrow(2 \pi x)^{-\frac{1}{2}} e^{x}, x \rightarrow \infty$ for fixed $\nu$ gives rise to $\delta\left(r-r^{\prime}\right)$ in the form of a limiting normal distribution in $\frac{1}{\sqrt{\tau}}\left(r-r^{\prime}\right)$ (roughly, since the series involves sums over $\nu \rightarrow \infty$ ).

The above result was obtained differently by Sommerfeld [1894]. It has been addressed within the context of default correlation by Zhou [2001]. The total probability of survival can be obtained by integrating over $x_{1}>0$ and $x_{2}>0$,

$$
p(\boldsymbol{x}, \tau)=\sqrt{\frac{2 \pi}{\tau}} \frac{r}{\varphi} e^{-\frac{r^{2}}{4 \tau}} \sum_{\nu \text { odd }} \frac{1}{\nu}\left[I_{\frac{\nu+1}{2}}\left(\frac{r^{2}}{4 \tau}\right)+I_{\frac{\nu-1}{2}}\left(\frac{r^{2}}{4 \tau}\right)\right] \sin (\nu \theta),
$$

where by $\nu$ odd, it is meant that the integers $k$ in (11) are restricted to be odd. This result is a special case of the general one (discussed in the next section) obtained differently by DeBlassie [1987].

\section{Many Particles}

We now come to a correlated system of $n$ particles with a position vector $\boldsymbol{x}$ describing collectively their positions governed by

$$
\frac{\partial f}{\partial \tau}=\frac{1}{2} \sum_{i j} R_{i j} \frac{\partial^{2} f}{\partial x_{i} \partial x_{j}}
$$

This is the differential equation for the transition probability density $\frac{1}{\sqrt{\operatorname{det} R}} f\left(\boldsymbol{x}, \boldsymbol{x}^{\prime}, \tau\right)$. More generally, one would have a covariance matrix, say $\Sigma_{i j}$, with the diagonal elements $\sigma_{i}=$ $\sqrt{\Sigma_{i i}}$. For convenience, $x_{i}$ 's are measured in units of $\sigma_{i}, i=1, \cdots, n$ so that $\Sigma_{i j}$ has been replaced by the correlation matrix $R_{i j}=\frac{1}{\sigma_{i} \sigma_{j}} \Sigma_{i j}$. Constant drift terms have also been ignored for simplicity.

The domain $D^{n}$ we are concerned with for $\boldsymbol{x}$ is $x_{i} \geq 0, i=1, \cdots, n$ with boundary conditions $f\left(\boldsymbol{x}, \boldsymbol{x}^{\prime}, \tau\right)=0$ when any one of the $x_{i}$ 's is set to zero. It is also expected that $f\left(\boldsymbol{x}, \boldsymbol{x}^{\prime}, \tau\right)$ goes to zero when any one of the $x_{i}$ 's is taken to infinity. As before, it is convenient to work in the diagonalized system that diagonalizes $R$ and scales it into identity so that the differential equation involves the Laplacian $\nabla^{2}$,

$$
\frac{\partial f}{\partial \tau}=\frac{1}{2} \nabla^{2} f, \quad \nabla^{2}=\sum_{i} \frac{\partial^{2}}{\partial y_{i}^{2}}
$$

This is the heat equation or the diffusion equation in $n$-dimensions. Dot-products defined such as $\boldsymbol{u} \cdot \boldsymbol{v}=\sum_{i j} R_{i j}^{-1} u_{i} v_{j}$ for any two vectors $\boldsymbol{u}$ and $\boldsymbol{v}$, and the implied lengths remain invariant but now get diagonalized expressions. It is further convenient to split the coordinates $\boldsymbol{y}$ into radial and angular parts, $r$ and $\hat{\boldsymbol{r}}$,

$$
r^{2}=\sum_{i} y_{i}^{2}=\sum_{i j} R_{i j}^{-1} x_{i} x_{j}, \quad \hat{\boldsymbol{r}}=\frac{\boldsymbol{y}}{r} .
$$


In the diagonalized system, unit radial vectors $\hat{\boldsymbol{r}}$ trace out a $n$-1-dimensional sphere $S^{n-1}$ at $r^{2}=1$ and domain $D^{n}$ intersects into a domain $\Omega^{n-1}$ in $S^{n-1}$.

A $\nu$-degree homogeneous function of $\boldsymbol{x}$ expressed as $r^{\nu} h_{\nu \sigma}(\hat{\boldsymbol{r}})$ defines a zero-degree homogeneous function $h_{\nu \sigma}(\hat{\boldsymbol{r}})$, with $\sigma$ labeling any multiplicity. Such a function solving the Laplace equation $\nabla^{2}\left(r^{\nu} h_{\nu \sigma}\right)=0$ in $D^{n}$ gives rise to an eigenvalue system ${ }^{1}$

$$
\nabla_{S}^{2} h_{\nu \sigma}(\hat{\boldsymbol{r}})=-\lambda h_{\nu \sigma}(\hat{\boldsymbol{r}})
$$

where $\lambda=\nu(\nu+n-2)$ and $\nabla_{S}^{2}=r^{2} \nabla^{2} . \nabla_{S}^{2}$ acting on functions of $\hat{\boldsymbol{r}}$ is the Laplacian on $S^{n-1}$ so that above defines a boundary value problem for the Dirichlet Laplacian on $\Omega^{n-1}$. Boundary value problems of this kind have been extensively studied and it turns out that the eigenvalues are all non-negative and discrete and that the eigenfunctions form a complete system. Hence $\nu$ 's can also be taken to be non-negative and discrete and we will assume that the eigenfunctions $h_{\nu \sigma}(\hat{\boldsymbol{r}})$ are normalized to form an orthonormal system

$$
\int_{\Omega^{n-1}} d^{n-1} \hat{r} h_{\nu \sigma}(\hat{\boldsymbol{r}}) h_{\nu^{\prime} \sigma^{\prime}}(\hat{\boldsymbol{r}})=\delta_{\nu \nu^{\prime}} \delta_{\sigma \sigma^{\prime}}
$$

where $d^{n-1} \hat{r}$ is the volume element (area element if $n=3$ ) on the unit sphere $S^{n-1}$.

The complete system of eigenfunctions $h_{\nu \sigma}(\hat{\boldsymbol{r}})$ enable us to expand $f\left(\boldsymbol{x}, \boldsymbol{x}^{\prime}, \tau\right)$ as

$$
f\left(\boldsymbol{x}, \boldsymbol{x}^{\prime}, \tau\right)=\sum_{\nu \sigma} g_{\nu \sigma}(r, \tau) r^{\nu} h_{\nu \sigma}(\hat{\boldsymbol{r}}) .
$$

The Laplacian on $g_{\nu \sigma} r^{\nu} h_{\nu \sigma}$ separates into that on $g_{\nu \sigma} r^{\nu}$ and $h_{\nu \sigma}$. Its action on $h_{\nu \sigma}$ is given by (19) so that the differential equation for $f\left(\boldsymbol{x}, \boldsymbol{x}^{\prime}, \tau\right)$ gives rise to

$$
\frac{\partial g_{\nu \sigma}}{\partial \tau}=\frac{1}{2} \frac{\partial^{2} g_{\nu \sigma}}{\partial r^{2}}+\frac{2 \nu+n-1}{2 r} \frac{\partial g_{\nu \sigma}}{\partial r} .
$$

This is again the differential equation describing the Bessel process. Hence, $\frac{r^{\prime 2}}{\tau}$ is distributed as the non-central chi-squared distribution with $2 \nu+n$ degrees of freedom and non-centrality parameter $\frac{r^{2}}{\tau}$. We thus have for the $r^{\prime}$-distribution,

$$
g_{\nu \sigma}(r, \tau) \propto \frac{1}{\tau} \chi^{2}\left(\frac{r^{\prime 2}}{\tau}, 2 \nu+n, \frac{r^{2}}{\tau}\right) \propto \frac{1}{\tau} e^{-\frac{1}{2 \tau}\left(r^{2}+r^{\prime 2}\right)} r^{-\nu-\frac{n-2}{2}} I_{\nu+\frac{n-2}{2}}\left(\frac{r r^{\prime}}{\tau}\right) .
$$

where again $I_{\nu}$ is the modified Bessel function. Some factors involving $r^{\prime}$ have been dropped as the appropriate normalization is determined below. Putting these together, we have

$$
f\left(\boldsymbol{x}, \boldsymbol{x}^{\prime}, \tau\right)=\frac{1}{\tau}\left(r r^{\prime}\right)^{-\frac{n-2}{2}} e^{-\frac{1}{2 \tau}\left(r^{2}+r^{\prime 2}\right)} \sum_{\nu} I_{\nu+\frac{n-2}{2}}\left(\frac{r r^{\prime}}{\tau}\right) \sum_{\sigma} h_{\nu \sigma}(\hat{\boldsymbol{r}}) h_{\nu \sigma}\left(\hat{\boldsymbol{r}}^{\prime}\right) .
$$

To verify the factors, note that the integration measure is $d^{n} x=\sqrt{\operatorname{det} R} r^{n-1} d r d^{n-1} \hat{r}$, and that $f\left(\boldsymbol{x}, \boldsymbol{x}^{\prime}, \tau\right) \rightarrow \sqrt{\operatorname{det} R} \delta\left(\boldsymbol{x}-\boldsymbol{x}^{\prime}\right)$ in the limit $\tau \rightarrow 0$. The asymptotic behavior $I_{\nu}(x) \rightarrow$ $(2 \pi x)^{-\frac{1}{2}} e^{x}, x \rightarrow \infty$ for fixed $\nu$ gives rise to $\delta\left(r-r^{\prime}\right)$ in the form of a limiting normal distribution in $\frac{1}{\sqrt{\tau}}\left(r-r^{\prime}\right)$ (roughly, since the series involves sums over $\nu \rightarrow \infty$ ).

The above result was obtained differently under different contexts by various authors. For $n=2$ it was obtained by Sommerfeld [1894]. For $n=3$, it was considered within the

\footnotetext{
${ }^{1}$ Laplacian acting on a product $g(r) h(\hat{\boldsymbol{r}})$ separates into $\left(\nabla^{2} g\right) h+g\left(\nabla^{2} h\right)$ when $h(\hat{\boldsymbol{r}})$ is zero-degree homogeneous function in $\boldsymbol{y}$ because of the vanishing of the cross term $(\nabla g) \cdot(\nabla h)=\partial_{r}(g) r^{-1}(\boldsymbol{y} \cdot \nabla) h=0$.
} 
context of circular cones by Carslaw and Jaeger [1959]. For general dimensions, it has been presented by Cheeger [1983]. The leading term in the series (24) can be obtained by making use of the expansion for the Bessel functions

$$
f\left(\boldsymbol{x}, \boldsymbol{x}^{\prime}, \tau\right) \sim \frac{2}{\Gamma\left(\nu_{1}+\frac{n}{2}\right)(2 \tau)^{\frac{n}{2}}}\left(\frac{r r^{\prime}}{2 \tau}\right)^{\nu_{1}} e^{-\frac{1}{2 \tau}\left(r^{2}+r^{\prime 2}\right)} h_{\nu_{1}}(\hat{\boldsymbol{r}}) h_{\nu_{1}}\left(\hat{\boldsymbol{r}}^{\prime}\right) .
$$

where $\nu_{1}$ is the first $\nu$ and $\Gamma$ is the Gamma function. In the case of an independent collection of particles in the presence of the barrier, we know that $f\left(\boldsymbol{x}, \boldsymbol{x}^{\prime}, \tau\right)$ is given by the product of individual expressions (3) so that

$$
f\left(\boldsymbol{x}, \boldsymbol{x}^{\prime}, \tau\right)=\left(\frac{2}{\pi \tau}\right)^{\frac{n}{2}} e^{-\frac{1}{2 \tau}\left(r^{2}+r^{\prime 2}\right)} \prod_{i=1}^{n} \sinh \left(\frac{x_{i} x_{i}^{\prime}}{\tau}\right) .
$$

In this case, series (24) can be viewed as a representation of power of sinh's in terms of modified Bessel functions.

The total probability of survival can be obtained by integrating $f\left(\boldsymbol{x}, \boldsymbol{x}^{\prime}, \tau\right)$ with respect to $\boldsymbol{x}^{\prime}$ on $D^{n}$ giving

$$
p(\boldsymbol{x}, \tau)=\tau^{\frac{n}{2}} r^{-n} e^{-\frac{r^{2}}{2 \tau}} \sum_{\nu} \widetilde{I}_{\nu+\frac{n-2}{2}}\left(\frac{r^{2}}{\tau}\right) \sum_{\sigma} h_{\nu \sigma}(\hat{\boldsymbol{r}}) \widetilde{h}_{\nu \sigma}
$$

where

$$
\widetilde{I}_{\nu}(a)=\int_{0}^{\infty} d t t^{\frac{n}{2}} e^{-\frac{t^{2}}{2 a}} I_{\nu}(t), \quad \text { and } \quad \widetilde{h}_{\nu \sigma}=\int_{\Omega^{n-1}} d^{n-1} \hat{r} h_{\nu \sigma}(\hat{\boldsymbol{r}})
$$

This result in terms of a hypergeometric function was obtained directly from the differential equation by DeBlassie [1987] who also discussed its implications for hitting times. The first term in the series is guaranteed to be positive since it is well known that the first $h_{\nu \sigma}$ can be taken to be positive within the domain. For large $\tau, p(\boldsymbol{x}, \tau)$ has the behavior $\sim \tau^{-\frac{\nu_{1}}{2}}$, implying that the expected hitting time will be finite if $\nu_{1}>2$. As discussed in the next section, for an independent collection of particles, $\nu_{1}=n$ so that the expected hitting time will be finite for $n \geq 3$. For a positively correlated collection of particles we expect $\nu_{1}<n$ but greater than $n-1$ as long as correlations are not too large so that the expected hitting time will be finite for $n \geq 3$. For a homogeneous collection of 3 particles with a common correlation of $\frac{1}{2}$, the numerical result of Ratzkin and Treibergs [2009] is applicable giving $\nu_{1} \approx 1.826$ and as discussed by them the expected hitting time will be finite only for $n \geq 4$.

\section{Spectrum On The Sphere}

The solution for the transition probability density obtained in the last section is expressed in terms of the eigenvalues and the eigenfunctions of the Laplacian on the sphere. Hence, let us have a look into spectrum of the Laplacian on a domain $\Omega^{n-1}$ on the sphere $S^{n-1}$ in $n$-dimensions corresponding to a collection of $n$ particles.

Many results are known in general about the eigenvalues and eigenfunctions of the Laplacian. For instance, the first eigenvalue has no multiplicity and the corresponding eigenfunction can be taken to be positive within the domain. In the case of independent particles in the absence of the barrier, the relevant domain on the sphere is the whole of $S^{n-1}$. In this case it is well known that $\nu$ is an integer taking values $0,1, \cdots, \infty$. The first 
$\nu$, denoted $\nu_{1}$, is zero corresponding to a constant function on $S^{n-1}$. The multiplicities of the eigenvalues are also known that will be revisited below.

In the independent case in the presence of the barrier, it is straightforward to show that $\nu_{1}=n$. In fact, being independent, the simplest homogeneous function solving the Laplace equation in $D^{n}$ and vanishing on the boundaries is of degree $n$ and is given simply by the product of the $n$-coordinates consistent with the equation (26). It is further clear that adding an independent particle to a correlated collection of particles would increase $\nu_{1}$ by one. If the added independent particle is not subject to the boundary condition, $\nu_{1}$ would of course remain the same. These observations are not trivial when formulated on the spherical domain.

To say more about the spectrum of the Laplacian on the sphere, let us next derive a 'generating function' $M(z)$ for the eigenvalues and the multiplicities in terms of $f\left(\boldsymbol{x}, \boldsymbol{x}^{\prime}, \tau\right)$. In the result (24), the multiplicity of eigenfunctions appears as a projection into the eigenspace. As we will see, the projection helps us in obtaining a generating function. Towards this end, setting $\boldsymbol{x}^{\prime}=\boldsymbol{x}$ and $\tau=1$ we have

$$
f(\boldsymbol{x}, \boldsymbol{x}, 1)=r^{2-n} e^{-r^{2}} \sum_{\nu} I_{\nu+\frac{n-2}{2}}\left(r^{2}\right) \sum_{\sigma}\left(h_{\nu \sigma}(\hat{\boldsymbol{r}})\right)^{2} .
$$

Note that a further operation of integrating over $\boldsymbol{x}$, along with any $\hat{r}$-independent weight, would integrate $\left(h_{\nu \sigma}(\hat{\boldsymbol{r}})\right)^{2}$ to unity (its normalization) introducing the multiplicity $m_{\nu}$. This procedure derives the following expression for the generating function (making use of the Laplace transform of $I_{\nu}$ ),

$$
M(z) \equiv \sum_{\nu} m_{\nu} z^{\nu}=\left(1-z^{2}\right) z^{-\frac{n}{2}} \int_{D^{n}} d^{n} x e^{-\frac{1}{2 z}(1-z)^{2} r^{2}} f(\boldsymbol{x}, \boldsymbol{x}, 1),
$$

where $0<z<1$ and $r$ is the length of $\boldsymbol{x}$. If the right side can be computed, this provides us with not only the multiplicities but the eigenvalues as well (from the exponents of $z$ ).

The above generating function arises naturally from the solution of the heat equation on the cone ${ }^{2}$. Its derivation does not assume a specific character of the domain, except that $D^{n}$ is conical intersecting $S^{n-1}$ into some domain $\Omega^{n-1}$. But its applicability depends on our knowledge of $f(\boldsymbol{x}, \boldsymbol{x}, 1)$. This is not expected to be the case in general. Below, let us first consider some special cases for which we do know $f(\boldsymbol{x}, \boldsymbol{x}, 1)$.

Consider again the case of independent particles with no barrier. In this case the integration range covers all of $\boldsymbol{x}$, that is, it includes $\boldsymbol{x}<0$ as well. Knowing $f(\boldsymbol{x}, \boldsymbol{x}, 1)$ as a product from $n$-individual free Brownian motions, one readily obtains

$$
M(z)=(1+z)(1-z)^{1-n}=\sum_{k=0}^{\infty} \frac{n+2 k-2}{n-2}\left(\begin{array}{c}
n+k-3 \\
k
\end{array}\right) z^{k}
$$

This gives the right eigenvalues and multiplicities on the whole sphere $S^{n-1}$. For the case of independent particles with the barrier, $f(\boldsymbol{x}, \boldsymbol{x}, 1)$ is given by $(26)$ that generates

$$
M(z)=z^{n}\left(1-z^{2}\right)^{1-n}=\sum_{k=1}^{\infty}\left(\begin{array}{c}
n+k-3 \\
k-1
\end{array}\right) z^{n+2 k-2} .
$$

\footnotetext{
${ }^{2}$ It is different from the usually studied trace of the heat kernel, $\operatorname{Tr}\left(e^{t \nabla_{S}^{2}}\right)$. It is not the eigenvalues $\nu(\nu+n-2)$ of $-\nabla_{S}^{2}$ that appear in the exponents, but rather $\nu$ 's themselves.
} 
In the $n=2$ case of two correlated particles, we have the result of section 2 that the $\nu$ 's are multiples of $\frac{\pi}{\varphi}$ and are all of multiplicity one. Its generating function is hence $z^{\frac{\pi}{\varphi}}\left(1-z^{\frac{\pi}{\varphi}}\right)^{-1}$ that becomes $z^{2}\left(1-z^{2}\right)^{-1}$ in the independent case in agreement with (32).

Note that $M(z)$, except for the factor $1-z^{2}$, factorizes across independent subsystems. Hence, $M(z)$ for a system comprising of two independent subsystems with generating functions $M_{1}(z)$ and $M_{2}(z)$ respectively is given by

$$
M(z)=\frac{1}{1-z^{2}} M_{1}(z) M_{2}(z)
$$

For example, if $p$ particles have no barrier and $q$ ones do, the product system has

$$
M(z)=\left(1-z^{2}\right)(1-z)^{-p} z^{q}\left(1-z^{2}\right)^{-q} .
$$

This corresponds to $n=p+q$ and the domain $\Omega^{n-1}$ on $S^{n-1}$ is obtained by cutting away the sphere through the center perpendicularly $q$-times. If there are correlated pairs particles, one or more of such pairs can be included in the above expression.

If we are interested in exploring the $h_{\nu \sigma}(\hat{\boldsymbol{r}})$ functions themselves, we could rederive our results without the angular integration to obtain

$$
M\left(\hat{\boldsymbol{r}}, \hat{\boldsymbol{r}}^{\prime}, z\right) \equiv \sum_{\nu} m_{\nu}\left(\hat{\boldsymbol{r}}, \hat{\boldsymbol{r}}^{\prime}\right) z^{\nu}=\left(1-z^{2}\right) z^{-\frac{n}{2}} \int_{0}^{\infty} d r r^{n-1} e^{-\frac{1}{2 z}(1-z)^{2} r^{2}} f\left(r \hat{\boldsymbol{x}}, r \hat{\boldsymbol{x}}^{\prime}, 1\right),
$$

where

$$
m_{\nu}\left(\hat{\boldsymbol{r}}, \hat{\boldsymbol{r}}^{\prime}\right)=\sum_{\sigma} h_{\nu \sigma}(\hat{\boldsymbol{r}}) h_{\nu \sigma}\left(\hat{\boldsymbol{r}}^{\prime}\right)
$$

This provides us with a generating function for the projections on to the eigenspaces. For an independent collection of $n$ particles without the barrier, that is on the whole sphere $S^{n-1}$, this gives

$$
M\left(\hat{\boldsymbol{r}}, \hat{\boldsymbol{r}}^{\prime}, z\right)=\frac{1}{\left|S^{n-1}\right|} \frac{\left(1-z^{2}\right)}{\left(1-2 z \cos \theta+z^{2}\right)^{\frac{n}{2}}},
$$

where $\theta$ is the angle between $\hat{\boldsymbol{r}}$ and $\hat{\boldsymbol{r}}^{\prime}$, and $\left|S^{n-1}\right|$ is the size of the sphere $S^{n-1}$. Other cases do not lead to simple expressions.

\section{Analytical Properties}

On continuing from the $z<1$ region, generating function $M(z)$ exhibits a singularity at $z=1$. At least for the various cases considered, the singularity is a pole of order $n-1$ (the dimension of the sphere) so that we may write around $z=1$

$$
M(z)=\frac{c_{n-1}}{(1-z)^{n-1}}+\frac{c_{n-2}}{(1-z)^{n-2}}+\cdots .
$$

Coefficients $c_{n-1}$ and $c_{n-2}$ can be determined,

$$
c_{n-1}=2 \frac{\left|\Omega^{n-1}\right|}{\left|S^{n-1}\right|}, \quad c_{n-2}=-\frac{1}{2} c_{n-1}-\frac{1}{2} \frac{\left|\partial \Omega^{n-1}\right|}{\left|S^{n-2}\right|} .
$$

It is convenient to write $c_{n-2}=-\frac{1}{2}(1+\gamma) c_{n-1}$ introducing

$$
\gamma=-2 \frac{c_{n-2}}{c_{n-1}}-1=\frac{1}{2} \frac{\left|S^{n-1}\right|}{\left|S^{n-2}\right|} \frac{\left|\partial \Omega^{n-1}\right|}{\left|\Omega^{n-1}\right|} .
$$


Above, $\left|\Omega^{n-1}\right|$ is the size of the domain $\Omega^{n-1}$ and $\left|\partial \Omega^{n-1}\right|$ is that of its boundary $\partial \Omega^{n-1}$. $\left|S^{n-1}\right|$ and $\left|S^{n-2}\right|$ are the sizes of $n-1$ and $n-2$ dimensional spheres of unit radii respectively. Sizes of $\Omega^{n-1}$ and $\partial \Omega^{n-1}$ are measured in units set by the $n-1$ dimensional sphere $S^{n-1}$ of unit radius on which they reside.

The leading coefficient $c_{n-1}$ can be determined by letting $z \rightarrow 1$ in the expression for $M(z)$. Note that the exponential inside the integral would no longer provide the suppression as $r \rightarrow \infty$. As $r \rightarrow \infty$, the effect of the boundary becomes insignificant and $f(\boldsymbol{x}, \boldsymbol{x}, 1)$ tends to a constant $(2 \pi)^{-\frac{n}{2}}$ ( $n$ factors from $(2)$ at $x=x^{\prime}, \tau=1$ ). The integral is thus dominated by regions near $r=\infty$ where the angular integral contributes $\left|\Omega^{n-1}\right|$. This gives

$$
M(1-\epsilon) \sim 2 \epsilon \int_{0}^{\infty} d r r^{n-1} e^{-\frac{1}{2} \epsilon^{2} r^{2}} \frac{\left|\Omega^{n-1}\right|}{(2 \pi)^{\frac{n}{2}}}=2 \frac{\Gamma\left(\frac{n}{2}\right)}{2 \pi^{\frac{n}{2}}} \frac{\left|\Omega^{n-1}\right|}{\epsilon^{n-1}} .
$$

The factors in front can be identified as twice the inverse size of the sphere $S^{n-1}$.

The next coefficient $c_{n-2}$ can be determined by the method of images. To start with, note that the contribution to $M(z)$ coming from the source alone, denoted $M_{S}(z)$, makes an order $n-1$ contribution as well,

$$
M_{S}(z)=\frac{c_{n-1}}{2} \frac{1+z}{(1-z)^{n-1}}=\frac{c_{n-1}}{(1-z)^{n-1}}-\frac{1}{2} \frac{c_{n-1}}{(1-z)^{n-2}} .
$$

In the method of images, the heat source placed within the domain induces images across the boundary that cancel out the source effect on the boundary to ensure zero boundary condition. Since $f(\boldsymbol{x}, \boldsymbol{x}, 1)$ is evaluated at the source location itself, as $\boldsymbol{x}$ is varied, the source moves and the images follow the source. As $r \rightarrow \infty$ many of the images will recede away from the source. The leading contribution comes from the image brought closest to the source by taking the source close to the boundary. Its contribution is $\sim-(2 \pi)^{-\frac{n}{2}} e^{-2 x_{\perp}^{2}}$. Here $x_{\perp}$ is the perpendicular distance of the source to the boundary so that the image to source distance is $2 x_{\perp}$. The contribution denoted $M_{I}(z)$ is

$$
M_{I}(1-\epsilon) \sim-\frac{2 \epsilon}{(2 \pi)^{\frac{n}{2}}} \int_{0}^{\infty} d r r^{n-2} e^{-\frac{1}{2} \epsilon^{2} r^{2}} \int_{\partial \Omega \perp} d x_{\perp} e^{-2 x_{\perp}^{2}}=-\frac{1}{2} \frac{\Gamma\left(\frac{n-1}{2}\right)}{2 \pi^{\frac{n-1}{2}}} \frac{\left|\partial \Omega^{n-1}\right|}{\epsilon^{n-2}} .
$$

The factors in front can be identified as half the inverse size of the sphere $S^{n-2}$.

Expansion (38) is a result of an expansion of $f(\boldsymbol{x}, \boldsymbol{x}, 1)$ in $r^{-1}$ in the expression (30) for $M(z)$. Since $\tau^{\frac{n}{2}} f(\boldsymbol{x}, \boldsymbol{x}, \tau)$ is function of the combination $\frac{r^{2}}{\tau}$, an expansion of $f(\boldsymbol{x}, \boldsymbol{x}, 1)$ in $r^{-1}$ is in fact an expansion of $f(\boldsymbol{x}, \boldsymbol{x}, \tau)$ in $\sqrt{\tau}$ at $\tau=1$. This is the well-known expansion of the heat kernel (see for instance Vassilevich [2003]), in our case on the cone $D^{n}$. Because the higher order terms of this expansion bring in more powers of $r$ into the denominator inside the integral in (30), it can only be used upto coefficient $c_{0}$. If the remainder falls off faster than $r^{-n}$ as $r \rightarrow \infty$, its integral will be finite at $z=1$ because of the $r \rightarrow 0$ behavior of $f(\boldsymbol{x}, \boldsymbol{x}, 1)$ evident from $(29)$. Also note here that the heat kernel expansion being an expansion in $r^{-1}$ does not see any terms of the type $e^{-r}$ for instance. That such terms are present can be seen by taking the example of $n=2$ independent system for which we know from (32) that $M(z)=z^{2}\left(1-z^{2}\right)^{-1}$. It turns out that in this case (30) is easily invertible to obtain

$$
\int_{\Omega^{1}} d \hat{r} f(\boldsymbol{x}, \boldsymbol{x}, 1)=\frac{1}{4}-\frac{1}{2} I_{0}\left(r^{2}\right) e^{-r^{2}}+\frac{1}{4} e^{-2 r^{2}},
$$

where $I_{0}$ is the modified Bessel function of order zero (this can also be obtained directly from the solution (14)). The first two terms on the right hand side give rise to the heat 
kernel expansion while the last term, not visible to the heat kernel asymptotics, is required for the $r \rightarrow 0$ behavior. Expansion (38) can also be obtained from the heat kernel expansion on $\Omega^{n-1}$ on the sphere itself using the identity

$$
M\left(e^{-s}\right)=\frac{s e^{q s}}{2 \sqrt{\pi}} \int_{0}^{\infty} \frac{d t}{t^{\frac{3}{2}}} e^{-q^{2} t-\frac{s^{2}}{4 t}} \operatorname{Tr}\left(e^{t \nabla_{S}^{2}}\right)
$$

where $\operatorname{Tr}$ refers to trace and $q=\frac{1}{2}(n-2)$. Analogous relation can be written down for the pointwise object $M\left(\hat{\boldsymbol{r}}, \hat{\boldsymbol{r}}^{\prime}, z\right)$. Inverse relations can be obtained by re-expressing them as Laplace transforms.

The series expansion of the kind at the $z=1$ pole are useful in estimating the growth of the spectrum at large eigenvalues. This is done with the help of a counting function

$$
W(\nu)=\sum_{\nu^{\prime}} m_{\nu^{\prime}} 1_{\nu^{\prime} \leq \nu}
$$

where $1_{\nu^{\prime} \leq \nu}$ is the step-function. $W(\nu)$ counts the eigenvalues, including multiplicity, up to $\nu$. Its Laplace transform is

$$
\widetilde{W}(s)=\int_{0}^{\infty} d \nu W(\nu) e^{-s \nu}=\frac{1}{s} M\left(e^{-s}\right) .
$$

As we have noted, $M\left(e^{-s}\right)$ is expected to have a pole of order $n-1$ at $s=0$. Here it should arise from the large $\nu$ behavior of $W(\nu)$. One finds

$$
W(\nu) \sim c_{n-1} \frac{\nu^{n-1}}{(n-1) !}+\frac{1}{2}(n-2-\gamma) c_{n-1} \frac{\nu^{n-2}}{(n-2) !}+\cdots, \quad \nu \rightarrow \infty .
$$

Expressed in terms of the eigenvalues $\lambda=\nu(\nu+n-2) \sim \nu^{2}$ of the Laplacian on $\Omega^{n-1}$, this is consistent with the Weyl scaling law (true for more general domains).

One may make an observation here on the analytically continued $M(z)$. Result (30) indicates naively a relation $M\left(z^{-1}\right)=-z^{n-2} M(z)$. However, this is not expected to hold as an approach to $z^{-1}$ from $z$ along the real axis encounters the singularity at $z=1$ (the situation is somewhat analogous to the Laplace transform of the modified Bessel function $I_{\nu}$ into $\left.\frac{1}{2 z}\left(1+z^{2}\right)\right)$. The various cases considered earlier suggest that a duality relation, if one exists, might instead be, for some $\gamma$,

$$
M\left(z^{-1}\right)=(-1)^{n-1} z^{n-2-\gamma} M(z)
$$

or equivalently, that $(1-z)^{n-1} z^{-\frac{1}{2}(1+\gamma)} M(z)$ is an even function of $\ln z$. To be consistent with the product formula (33), $\gamma$ should be additive across independent subsystems. Consistency with the series (38) implies that $\gamma$ is in fact the one introduced in (40). Consistency further relates the even coefficients of the series $c_{n-2}, c_{n-4}, \cdots$ to the odd ones $c_{n-1}, c_{n-3}, \cdots$. This of course needs to agree with the heat kernel expansion. For the various cases considered earlier, relation (49) holds and $\gamma$ is in fact $\nu_{1}$, and the expression (40) for $\gamma$ reproduces $\nu_{1}$ 's. Because $\nu_{1}$ is additive across independent subsystems, this will also hold for domains factorizable into such cases. 


\section{A Scaling Procedure}

Domain $\Omega^{n-1}$ on the sphere $S^{n-1}$ for a correlated system $n$ particles is closely related to domain $\Omega_{0}^{n-1}$ corresponding to the independent case. Domain $\Omega_{0}^{1}$ for the case of 2 independent particles is given by the quadrant circular arc considered in section 2. Domain $\Omega_{0}^{2}$ for the case of 3 independent particles is given by the octant triangle on the twodimensional sphere, a triangular region having three 90 degree angles taking up one eighth of the spherical surface. It can be viewed as an extension of $\Omega_{0}^{1}$ into the third dimension. Domains $\Omega_{0}^{n-1}$ in higher dimensions can be similarly approached.

For a positively correlated collection of particles, domain $\Omega^{n-1}$ tends to be larger compared to $\Omega_{0}^{n-1}$. This can be verified in the case of a homogeneous collection with a single correlation parameter. It is a result that the eigenvalues of the Laplacian do not increase as the domain is enlarged. Hence, we expect $\nu \leq n$ for a positively correlated collection. These observations suggest that we may look for a scaling factor to estimate the eigenvalues in the correlated system. Applying scaling to the eigenvalues of the Laplacian itself, as is usually done, turns out to be not satisfactory. Let us hence look for a generating function $M(z)$ on the domain $\Omega^{n-1}$ of the form

$$
M(z)=z^{\alpha} M_{0}\left(z^{\beta}\right),
$$

where $M_{0}(z)$ is the known generating function on the domain $\Omega_{0}^{n-1}$. Scaling factors $\alpha$ and $\beta$ can be determined by expanding $M(z)$ and $M_{0}(z)$ into their series (38) at $z=1$ and matching the first two coefficients (39) for the two domains. In terms of the eigenvalues, (50) implies that, given the eigenvalues $\lambda_{0 k}=\nu_{0 k}\left(\nu_{0 k}+n-2\right), k=1,2, \cdots$ of the Laplacian on $\Omega_{0}^{n-1}$, the eigenvalues $\lambda_{k}=\nu_{k}\left(\nu_{k}+n-2\right)$ on $\Omega^{n-1}$ can be estimated according to

$$
\nu_{k}=\alpha+\beta \nu_{0 k}, \quad k=1,2, \cdots,
$$

where $\alpha$ and $\beta$ obtained from the coefficients (39) are

$$
\alpha=\frac{1}{2}\left[\gamma-\beta \gamma_{0}+(\beta-1)(n-2)\right], \quad \beta=\left[\frac{\left|\Omega_{0}^{n-1}\right|}{\left|\Omega^{n-1}\right|}\right]^{\frac{1}{n-1}} .
$$

$\gamma$ and $\gamma_{0}$ are as given by (40) for the two domains $\Omega^{n-1}$ and $\Omega_{0}^{n-1}$ respectively. Expression for $\alpha$ can also be derived directly from the duality relation (49) though such a relation is not a requirement. If $\Omega^{n-1}$ and $\Omega_{0}^{n-1}$ are closely related and the eigenvalues are well separated, multiplicities are likely to remain the same. Eigenfunctions will of course be different; perhaps there is a scaling procedure for them as well.

The above procedure requires computing the domain sizes $\left|\Omega^{n-1}\right|$ and $\left|\partial \Omega^{n-1}\right|$. For a correlated system, $\left|\Omega^{n-1}\right|$ can be computed as

$$
\left|\Omega^{n-1}\right|=\frac{\left|\Omega_{0}^{n-1}\right|}{\sqrt{\operatorname{det} R}}\left(\frac{2}{\pi}\right)^{\frac{n}{2}} \int_{0}^{\infty} d^{n} x e^{-\frac{1}{2} x^{T} R^{-1} x} .
$$

For $\Omega_{0}^{n-1}$ an 'octant triangle', $\left|\Omega_{0}^{n-1}\right|$ is $2^{-n}$ of that of the sphere. $\left|\partial \Omega^{n-1}\right|$ can be computed with the same formula with $R^{-1}$ restricted to one dimension less. An example of a correlated system is a homogeneous collection of particles with a single correlation parameter $\rho$ such that the correlation matrix is

$$
R_{i j}=(1-\rho) \delta_{i j}+\rho, \quad R_{i j}^{-1}=\frac{1}{1-\rho} \delta_{i j}-\frac{\rho}{(1-\rho)(1+(n-1) \rho)} .
$$


This matrix has determinant $\operatorname{det} R=(1-\rho)^{n-1}(1+(n-1) \rho)$. Diagonalization to coordinates to $y_{i}$ can be carried out for instance by

$$
\begin{aligned}
& x_{i}=a y_{i}+b \sum_{j=1}^{n} y_{j}, \quad y_{i}=\frac{1}{a} x_{i}-\frac{b}{a(a+n b)} \sum_{j=1}^{n} x_{j}, \\
& a=\sqrt{1-\rho}, \quad b=\frac{1}{n}(\sqrt{1+(n-1) \rho}-\sqrt{1-\rho}) .
\end{aligned}
$$

For this homogeneous system, the domain size expression (53) simplifies to

$$
\left|\Omega^{n-1}\right|=\left|S^{n-1}\right| \int_{-\infty}^{\infty} \frac{d u}{\sqrt{2 \pi}} e^{-\frac{1}{2} u^{2}}\left[N\left(\frac{\sqrt{\rho} u}{\sqrt{1-\rho}}\right)\right]^{n},
$$

where $N$ is the cumulative standard normal distribution function. The same expression upon setting $n \rightarrow n-1$ and $\rho \rightarrow \frac{\rho}{1+\rho}$ gives $\frac{1}{n}\left|\partial \Omega^{n-1}\right|$. The expression can be evaluated for $n=2$ giving $\Omega^{1}=\cos ^{-1}(-\rho)$ in agreement with section 1 . It can also be evaluated for $\rho=\frac{1}{2}$ for any $n$ giving $\left|\Omega^{n-1}\right|=\frac{1}{n+1}\left|S^{n-1}\right|$ corresponding to a domain on $S^{n-1}$ analogous to a tetrahedral triangle on the two-sphere. The integral is an increasing function of $\rho$ so that $\left|\Omega^{n-1}\right|>\left|\Omega_{0}^{n-1}\right|$ for $\rho>0$. As $\rho \rightarrow 1$ it tends to cover half the sphere. For very small $\rho$, the integral is $\approx 2^{-n}\left(1+\frac{1}{\pi} n(n-1) \rho\right)$ so that $\alpha \approx-\frac{1}{\pi} n(n-2) \rho, \beta \approx 1-\frac{1}{\pi} n \rho$ and the first eigenvalue corresponds to $\nu_{1} \approx n-\frac{2}{\pi} n(n-1) \rho$.

\section{Numerical Comparisons}

Ratzkin and Treibergs [2009] have studied a capture problem that can be recast into that of a homogeneous collection of correlated particles having $\rho=\frac{1}{2}$. The authors present a theoretical and numerical framework and compute the first eigenvalue $\lambda_{1}=\nu_{1}\left(\nu_{1}+n-2\right)$ of the Laplacian on the tetrahedral triangle on the two-sphere $S^{2}$ of unit radius. To find out how good our estimate is, let us compare their result 5.159 to that of the scaling procedure. For the domain $\Omega_{0}^{2}$, choose the octant triangle having $\left|\Omega_{0}^{2}\right|=\frac{\pi}{2}$ and $\left|\partial \Omega_{0}^{2}\right|=\frac{3 \pi}{2}$ so that $\gamma_{0}=\nu_{01}=3$. The tetrahedral triangle has $\left|\Omega^{2}\right|=\pi$ and $\left|\partial \Omega^{2}\right|=3 \cos ^{-1}\left(-\frac{1}{3}\right)$ so that $\gamma=\frac{3}{\pi} \cos ^{-1}\left(-\frac{1}{3}\right)$ and $\beta=\frac{1}{\sqrt{2}}$. This gives $\nu_{1}=1.826$ and $\lambda_{1}=5.162$ in excellent agreement with their result, indicating that the scaling procedure should be satisfactory for homogeneous collections.

A spherical cap is a circular domain on the two-sphere. If its radius relative to its center in angles is $\theta$, it has $\left|\Omega^{2}\right|=2 \pi(1-\cos \theta)$ and $\left|\partial \Omega^{2}\right|=2 \pi \sin \theta$. In this case $\Omega_{0}^{2}$ can be chosen to be the half sphere that has $M_{0}(z)=z(1-z)^{-2}, \gamma_{0}=\nu_{01}=1$. We then get

$$
\alpha=\frac{1}{2}\left(\cot \frac{\theta}{2}-1\right), \quad \beta=\frac{1}{\sqrt{2} \sin \frac{\theta}{2}} .
$$

As $\theta \rightarrow 0$, this leads to $\nu_{1} \theta \rightarrow 1+\sqrt{2}=2.4142$. This compares well with the flat disk solution $\nu_{1} \theta=j_{0,1}$ where $j_{0,1}=2.4048$ is the first zero of the Bessel function $J_{0}$.

The usual scaling procedure applied to the eigenvalues of the Laplacian itself is based on just the size of the domain, and hence is not able to differentiate the effects of the boundary. Ratzkin and Treibergs [2009] present a theoretical result $\lambda_{1}=4.936$ for the first eigenvalue on a spherical cap having the same area as the tetrahedral triangle (that is $\theta=\frac{\pi}{3}$ ). Scaling with (57) gives $\lambda_{1}=4.949$ in excellent agreement. 
A sector of the spherical cap making an angle $\varphi$ has $\left|\Omega^{2}\right|=\varphi(1-\cos \theta)$ and $\left|\partial \Omega^{2}\right|=$ $\varphi \sin \theta+2 \theta$. Choosing $\Omega_{0}^{2}$ to be such a sector on the hemisphere $\left(\theta=\frac{\pi}{2}\right)$ that has $M_{0}(z)=$ $z^{1+\frac{\pi}{\varphi}}\left(1-z^{\frac{\pi}{\varphi}}\right)^{-1}, \gamma_{0}=\nu_{01}=1+\frac{\pi}{\varphi}$, we get

$$
\alpha=\frac{1}{2}\left(\cot \frac{\theta}{2}+\frac{\theta}{\varphi \sin ^{2} \frac{\theta}{2}}-\frac{\pi}{\sqrt{2} \varphi \sin \frac{\theta}{2}}-1\right), \quad \beta=\frac{1}{\sqrt{2} \sin \frac{\theta}{2}} .
$$

Ratzkin and Treibergs [2009] present a theoretical result $\lambda_{1}=5.0046$ for the case $\varphi=\frac{2 \pi}{3}$ and $\theta=\cos ^{-1}\left(\frac{-1}{\sqrt{3}}\right)$ whereas the scaling procedure gives $\lambda_{1}=5.1046$.

The differences relative to the data, though small, are consistently on the higher side. Whether this is just a coincidence or whether the scaling procedure results in a very close upper bound is worth investigating. Better results could be possible with improved scaling relations in (51) involving more parameters to match other coefficients in the series (38) (implied from the heat kernel expansion). The scaling procedure very likely may not yield good results for all the eigenvalues, but its potential to do so for the first few is intriguing. Also interesting to study is the applicability of a similar scaling procedure for more general domains, other than those on spheres, by taking (45) as defining $M(z)$.

\section{References}

DeBlassie, R. D., (1987), "Exit times from cones in $\mathbb{R}^{n}$ of Brownian motion", Probability Theory and Related Fields. 74-1 (1987), 1-29.

Carslaw, H. S. and Jaeger, J. C., (1959), "Conduction of heat in solids", Oxford University Press, Oxford, 1959.

Cheeger, J., (1983), "Spectral geometry of singular Riemannian spaces", Journal of Differential Geometry 18-4 (1983), 575-657.

Ratzkin, J. and Treibergs, A., (2009), "A capture problem in Brownian motion and eigenvalues of spherical domains", Transactions of the American Mathematical Society 361 (2009), 391-405.

Sommerfeld, A., (1894), "Zur analytischen Theorie der Wärmeleitung", Mathematische Annalen 45-2 (1894), 263-277.

Vassilevich, D. V., (2003), "Heat kernel expansion: user's manual", Physics Reports, 388 (2003), 279-360.

Zhou, C., (2001), "An analysis of default correlations and multiple defaults", Review of Financial Studies, 14-2 (2001), 555-576. 